\title{
Classification des Systèmes Cobotiques
}

\section{Classification of Cobotic Systems}

\author{
Jean-Marc Salotti ${ }^{1,2}$, Eric Ferreri ${ }^{1}$, Olivier Ly ${ }^{3}$, David Daney ${ }^{2}$ \\ ${ }^{1}$ Laboratoire IMS, CNRS, Institut Polytechnique de Bordeaux, Université de Bordeaux, jean-marc.salotti@ensc.fr, \\ eric.ferreri@ensc.fr \\ ${ }^{2}$ Equipe Auctus, Iniria Bordeaux, david.daney@inria.fr \\ ${ }^{3}$ LABRI, CNRS, Université de Bordeaux, ly@labri.fr
}

RÉSUMÉ. La classification des systèmes cobotiques est difficile car il existe de nombreux critères pour décrire la collaboration homme robot. Après état de l'art et définition des termes, nous proposons d'étudier les flux d'informations entre le cobot, au sens le plus large du terme, l'humain, l'environnement, et l'interface afin de caractériser les différents types de collaboration. La représentation sous la forme d'un schème de l'interaction s'avère riche et pertinente. Dix exemples sont analysés et discutés. Plusieurs schèmes présentent des caractéristiques discriminantes qui permettent de qualifier les systèmes cobotiques. On distingue ainsi le système symbiotique, avec un échange constant d'informations et un partage efficace de la tâche, l'humain augmenté, la sous-traitance, l'assistance à l'effort et l'assistance intelligente.

ABSTRACT. The classification of cobotic systems is hard as there exist numerous criteria to describe human robot collaboration. After state of the art and definition of the main terms, we propose to study the flow of information among the cobot, in its broadest sense, the human, the environment and the interface in order to characterize the different types of collaboration. The description with a scheme of interaction seems particularly appropriate. Ten examples are considered and discussed. Several schemes present discriminant features that allow the qualification and naming of the cobotic systems. It is thus possible to identify the symbiotic system, with a constant information exchange and an efficient work sharing, the augmented human, the sous-traitance, the assistance to effort and the intelligent assistance.

MOTS-CLÉS. Cobotique, robotique collaborative, cobot.

KEYWORDS. Cobotic, collaborative robot, cobot.

\section{Introduction}

La collaboration hommes robots s'est fortement développée depuis les années 2000 (BRO 2004, BRO 2013, CLA 2013). Au Japon, aux Etats-Unis, en Allemagne, notamment, on entreprend l'étude et le développement de nombreux robots dont la vocation est d'interagir avec les humains, que ce soit avec les robots humanoïdes comme Asimo (HON 2001), les robots jouets comme AIBO (SON 2001), les robots simulant des émotions de type Kismet (BRE 2001), les robots militaires (Bigdog, UAV), les exosquelettes comme XOS, ou HULC (FER 2007, HER 2009), ou les robots d'assistance chirurgicale (LEE 2014). En 2004, Brooks écrivait (BRO 2004) : < I think that 15 years from now, robots will be everywhere, as e-mail and the Web are now. $>>$ Treize ans plus tard, on assiste en effet à une croissance très forte du secteur de la robotique et de la cobotique. En 2012, le gouvernement français tente d'attraper le train en marche avec la publication du rapport PIPAME et une série d'actions pour promouvoir la robotique (ERD 2012). Plus récemment, nous avons participé à un groupe de travail du Domaine d'Activité Stratégique (DAS) Interactions Hommes Systèmes du pôle Aerospace Valley, qui s'est intéressé à la cobotique pour répondre à des besoins industriels. Le cobot, ou robot collaborant avec l'humain, apparait désormais comme un outil indispensable pour les entreprises qui visent la modernisation de leurs ateliers pour répondre aux exigences d'efficacité et d'adaptabilité de ce qu'on appelle l'usine du futur (SMA 2013). La " cobotisation » est demandée, mais s'agit-il d'un effet de mode, ou bien d'un passage obligatoire pour résoudre de vrais problèmes industriels ? Pourquoi, comment, quelle efficacité, pour répondre à quels besoins, avec quel cobot ? Afin de participer à la réflexion sur le sujet, nous proposons d'aborder le difficile problème de la classification des systèmes cobotiques, sans nous restreindre à la problématique industrielle. Dans un premier temps, nous tentons de définir les concepts en rappelant les critères proposés dans la littérature pour classer les cobots ou les systèmes cobotiques. Du point de vue « cognitique », c'est l'analyse des interactions qui nous parait 
le meilleur angle d'approche. Quelles sont les informations échangées, à quel rythme, avec quel niveau d'abstraction, qui prend la décision et qui réalise l'action, ce sont les questions clés qui guident cette étude. Nous proposons des patterns d'interaction caractéristiques de certains systèmes cobotiques. En analysant les faiblesses et les forces de la machine et de l'humain et en tenant compte des différentes modalités de couplage, nous tentons de donner du sens aux interactions, afin de fournir quelques clés pour comprendre les enjeux et perspectives de ce domaine.

\section{Cobots et cobotique}

\subsection{Définition de la cobotique}

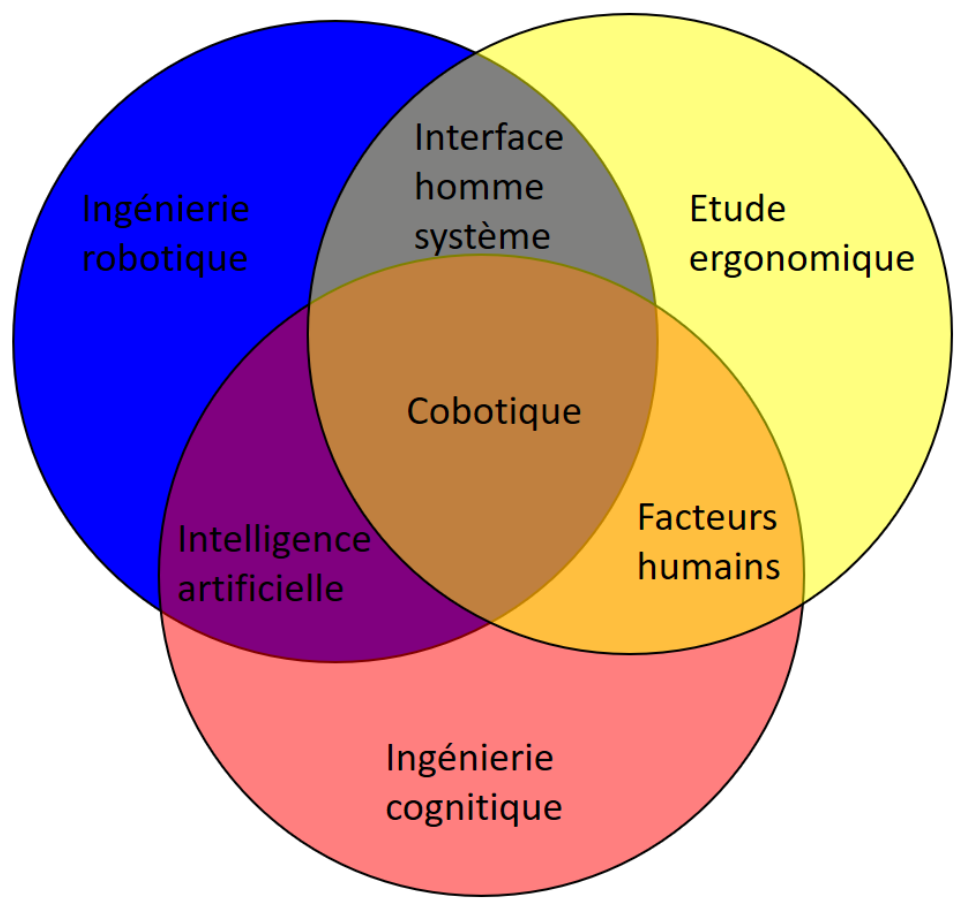

Figure 2.1. Les problématiques de cobotique sont au cœur de plusieurs domaines fondamentaux.

La cobotique est un nouveau domaine scientifique qui étudie les systèmes hommes robots collaboratifs, appelés systèmes cobotiques (CLA 2013). Le terme dérive du concept de cobot, proposé en 1996 par Colgate, Wannasuphoprasit et Peshkin (COL 1996, PES 1999). Les différents types d'interaction, leur efficacité, leur intensité, les différents moyens de perception, la prise de décision et les modalités d'action sont au cœur des problématiques de ce domaine. La cobotique est proche de la robotique. Elle n'englobe cependant pas toutes les problématiques de la robotique, car l'objet d'étude n'est pas le robot en lui-même, qui peut avoir des performances particulières en fonction de caractéristiques énergétiques, électroniques, mécaniques, ou automatiques qui ne revêtent a priori pas un grand intérêt ici. De manière symétrique, la robotique n'inclut pas la cobotique, car certains aspects, comme la représentation que l'humain se fait du robot, l'ergonomie du poste de travail ou l'acceptabilité par l'opérateur, relèvent des études ergonomiques et de l'ingénierie cognitique. La cobotique inclut également des questionnements et des études, qui concernent typiquement les interactions hommes robots, comme par exemple le degré et l'efficience du couplage, ou la dynamique spatio-temporelle de l'activité exercée par le système cobotique pris comme une entité unique. Du point de vue ensembliste, comme cela est illustré figure 2.1, la cobotique est donc à l'intersection de trois domaines, la robotique, l'ergonomie et la cognitique. Ses problématiques sont également proches de l'intelligence artificielle, de l'interface homme machine et des facteurs humains. En ce qui concerne le terme « cobot», il a été introduit pour désigner des robots industriels collaboratifs travaillant dans le même espace de travail que l'humain (PES 1999). Encore aujourd'hui, cette définition est souvent admise par les roboticiens, notamment dans le milieu industriel. Les drones, en particulier, sont 
rarement qualifiés de cobots. Cependant, du point de vue de la cognitique et des interactions hommes systèmes, cette restriction du domaine collaboratif (uniquement industriel) et du mode de collaboration (même espace de travail) parait arbitraire. Cette étude ne se limitant pas à la robotique industrielle, nous proposons d'élargir la définition pour englober tous les robots collaboratifs. La définition même de « robot » soulevant quelques difficultés, pour lever les ambiguïtés et distinguer en particulier les systèmes mécaniques des robots, nous proposons de définir le terme de robot comme un système mécatronique comportant un ensemble de capteurs, un corps tangible, un microcontrôleur programmable et un système moteur pourvu d'actionneurs pour la manipulation ou la locomotion. Ces deux fonctions sont incluses dans la définition afin de distinguer les robots des machines-outils. Un cobot étant défini comme un robot collaboratif, il convient de remarquer que la simple description matérielle ne suffit pas à déterminer la nature du robot. Il faut pour cela déterminer s'il collabore ou pas. Un robot conçu pour collaborer n'est donc qu'un cobot potentiel. La difficulté est de définir Notons également que, selon notre définition, une voiture est considérée comme un cobot.

\subsection{Classification en fonction du rôle de l'opérateur}

Les systèmes cobotiques sont très diversifiés et les applications sont nombreuses. De nombreux auteurs ont tenté de classer ces systèmes pour structurer le domaine (RAH 1990, PAR 2000, YAN 2004, WAL 2014, MOU 2015), avec des communautés spécialisées dans les problématiques sociales des interactions hommes systèmes (BAR 2004), ou dans les problématiques physiques et sécuritaires de ces mêmes interactions (BIC 2008, HAD 2007). On peut s'intéresser aux robots et les distinguer selon leur architecture, leur degré d'autonomie, leur mobilité, leur capacité de transport, de manipulation, leur taille, leur éloignement de l'opérateur. Il s'agit dans ce cas d'une classification qui omet totalement la composante humaine du système cobotique. A l'opposé, on peut classer les systèmes cobotiques en fonction du rôle de l'humain. Scholtz propose par exemple la distinction suivante (SCH 2003) :

- OPERATOR : Il opère ou téléopère le cobot, qui a en général une très faible autonomie décisionnelle, comme par exemple pour le Da Vinci ou le drone.

- COWORKER : Il travaille dans le même environnement que le robot, en parallèle, et a parfois quelques interactions avec lui, par exemple en lui prenant la pièce qu'il vient de traiter.

- SUPERVISOR : Il fournit des instructions au cobot et vérifie éventuellement la qualité du travail. On peut noter que les instructions doivent être fournies alors que le cobot est en fonctionnement. Dans le cas contraire, il s'agit plutôt d'une programmation ou d'un paramétrage, il n'y a pas de collaboration et le cobot est en réalité un robot.

- BYSTANDER : Il est present dans le même environnement que le robot et entre parfois dans sa zone de travail, sans toutefois avoir de réelle interaction avec lui. Le robot a par exemple un capteur de présence qui lui indique qu'un humain est dans sa zone et il passe automatiquement dans un mode plus lent ou s'arrête temporairement. La collaboration est réduite au strict minimum, mais on peut malgré tout parler de cobot car le mode opératoire dépend bien, de manière indirecte, de l'humain.

- MAINTENANCE OPERATOR : La maintenance ne concerne pas la cobotique.

- DESIGNER/PROGRAMMER : La programmation du robot ne concerne pas non plus la cobotique.

C'est une classification utile pour l'industrie, mais sans doute un peu restrictive. Dans le cas général, on ne peut résumer le rôle d'un humain à celui d'un « opérateur ». Selon le domaine d'application, il peut s'agir d'un visiteur, d'un patient, d'un militaire, d'un enfant, par exemples. Cette classification est cependant intéressante car elle caractérise implicitement le type d'interaction qui existe entre le cobot et l'humain. Toutefois, elle reste relativement superficielle car elle ne permet pas de prendre en compte 
le degré d'autonomie du cobot ou l'intensité du couplage, pour distinguer par exemple l'exoquelette et le robot télécommandé. Pour classer les systèmes cobotiques, il faudrait tenir compte des principales caractéristiques des interactions, tout en intégrant des informations robotiques ou humaines si elles sont essentielles. Il est bien entendu possible de multiplier les critères descriptifs et de les associer pour établir une classification plus pertinente, mais on perd alors en simplicité.

\subsection{Autonomie des cobots}

Le développement de l'intelligence artificielle a fortement augmenté la capacité des robots à résoudre des problèmes complexes, ce qui a permis d'augmenter leur autonomie et a enrichi les modalités d'interaction (WOO 1999, RUS 2016). Même si d'immenses progrès ont été réalisés dans des domaines réputés difficiles, comme la reconnaissance des formes, la navigation en terrain inconnu, le raisonnement dans l'incertain, la manipulation d'objets, ou la reconnaissance de la parole, les erreurs et les incertitudes sont encore trop importantes pour envisager des mises en œuvres simples et rapides pour des applications non triviales. Les approches de type deep learning, associées à des méthodes symboliques et des systèmes multi-agents sont prometteuses, mais la complexité des problèmes abordés nécessite la prise en compte de connaissances implicites et d'informations tirées du contexte, ce qu'on ne parvient pas à faire de manière efficace (THR 2005, RUS 2016). Selon Lemaignan, ces difficultés structurent la recherche autour de trois challenges : la communication homme-robot, l'action conjointe et l'explicitation de l'intention (LEM 2015). Ces approches sont généralement basées sur une architecture de contrôle qui permet un haut niveau d'abstraction, une planification fine des tâches et qui structure la collaboration.

\subsection{Applications de systèmes cobotiques}

Il est difficile de recenser les applications tant elles sont nombreuses et variées. En 2016, Sheridan, spécialiste facteurs humains, a proposé une revue de synthèse à partir de 4 applications relativement représentatives des travaux en interactions hommes robots (SHE 2016). Nous les rappelons ici :

1. Contrôle supervisé : Le rôle de l'humain est de commander et surveiller les actions du robot, par exemple pour aller chercher un objet à un emplacement donné, effectuer un empaquetage, etc. Une certaine autonomie est requise sur chaque tâche.

2. Commande à distance : il s'agit typiquement du cas des drones, que ce soit en environnement aérien, terrestre ou sous-marin, avec des tâches non triviales. En général, il y a peu d'autonomie, toutes les commandes viennent de l'humain.

3. Véhicule autonome : l'humain est considéré comme un passager dont le rôle se résume à définir la destination, par exemple pour un trajet autoroutier, ferroviaire ou en mode pilote automatique dans un avion.

4. Application avec interaction sociale : c'est le cas de la robotique de service, par exemple le robot d'assistance aux personnes âgées, ou le robot hôtesse d'accueil.

Cette taxonomie n'est pas représentative de la variété des cobots industriels, mais permet déjà de distinguer de grands ensembles.

\subsection{Interactions hommes robots}

De nombreux travaux sont basés sur un modèle des échanges d'information entre le robot et l'humain. Parfois, le modèle est plus complexe avec la modélisation de la tâche ou de l'environnement. Par exemple, Rahimi et Karwowski, soucieux des problématiques de sécurité et de facteurs humains posées par ces interactions, proposent dans leurs travaux fondateurs une modélisation en 5 éléments $(\mathrm{T}$, $\mathrm{U}, \mathrm{R}, \mathrm{E}, \mathrm{I})$, où $\mathrm{T}$ est la tâche, U l'utilisateur, $\mathrm{R}$ le robot, $\mathrm{E}$ l'environnement et I un ensemble d'interactions (RAH 1990). Les systèmes cobotiques étant par essence des systèmes collaboratifs, on 
peut tenter de les classer sur des critères liés à l'interaction entre l'homme et le robot. Yanco and Drury proposent par exemple de distinguer les différents types d'interaction et les interfaces associées (YAN 2004). Le capteur utilisé pour la perception du robot conditionne et structure la communication avec l'humain.

\section{Schème de l'interaction}

\subsection{Modélisation de l'interaction}

L'étude des flux d'information est fondamentale pour caractériser l'interaction. Intuitivement, en première approche, 3 grandes parties physiques semblent structurer celle-ci :

- L'humain H

- Le cobot $C$

- L'environnement E

Cependant, ces 3 parties ne sont pas toujours faciles à identifier. En effet, il existe souvent une interface, notamment entre l'humain et le cobot (e.g. un joystick et un écran), qui permet un échange d'informations. Selon le point de vue, cette interface peut appartenir au cobot ou à l'environnement. Dans le cas d'une télécommande et de lunettes de réalité augmentée, elle est éloignée du cobot, ce qui suggère qu'elle ne peut pas être incluse dans celui-ci. D'un point de vue fonctionnel, cependant, l'interface est souvent indispensable et doit être intégrée à chaque environnement applicatif. Etant donné son rôle particulier, nous proposons d'en faire une quatrième partie, appelée I.

Pour caractériser l'interaction, nous proposons d'expliciter les relations entre les 4 parties et leur propriétés générales :

- La première relation concerne le type d'information captée sur l'environnement ou échangée entre les parties. Les échanges peuvent concerner les données de l'environnement (cas du drone par exemple), des données du problème à résoudre, l'état courant de la tâche, ou des commandes directes. Les informations sont brutes et non traitées (une position, une image, un son, une commande), ou abstraites (le résultat d'un traitement complexe, un mouvement ou un geste à interpréter, un ordre vocal à reconnaitre). Le type d'information échangée étant étroitement corrélé à la capacité d'abstraction du cobot et à sa capacité à résoudre des problèmes complexes, cette relation permet implicitement, en première approximation, de caractériser le système cognitif de la machine.

- La deuxième relation concerne le type d'action appliquée par les effecteurs, soit sur l'environnement, soit de l'humain vers le cobot, soit du cobot vers l'humain. L'échange d'informations et l'action ont parfois une frontière floue. Par exemple, lorsque l'utilisateur appuie sur un bouton, il exerce une action, mais le bouton peut être considéré comme un élément du cobot ou de l'interface et il s'agit plus d'un moyen de communication avec le cobot qu'une action sur celui-ci. C'est cette interprétation que nous privilégierons. L'action est définie dans cette étude comme l'application d'une force d'intensité variable et dont le résultat ne se résume pas au changement binaire d'un état.

- La troisième variable est l'intensité du couplage homme / cobot. Elle est d'autant plus importante que les échanges ou les actions sont nombreux et fréquents entre les parties, notamment l'humain, le cobot et éventuellement l'interface. Un exosquelette a typiquement un couplage fort, car les échanges sont permanents, au contraire d'un cobot dont la tâche serait supervisée.

- Enfin, la quatrième variable concerne le cheminement de l'information, de la perception de l'environnement jusqu'à l'action. Certaines informations vont systématiquement de l'environnement au cobot, puis à l'humain et d'autres de l'humain au cobot, parfois selon un ordre et un rythme propre à l'application. Il est important de pouvoir expliciter ce cheminement. 
Remarque importante : ces quatre caractéristiques ne suffisent pas à décrire toutes les modalités et problématiques de l'interaction. Il faut y ajouter en particulier les objectifs et contraintes de la tâche, en termes de sécurité et performance, ainsi que tout le contexte de son exécution. Ce sont des éléments essentiels que nous avons mis en évidence dans une étude précédente (MOU 2016). Nous proposons ici de restreindre l'analyse et de nous focaliser sur les relations informationnelles existant entre les parties physiques.

\subsection{Etude de cas}

Pour mettre en application la modélisation, nous proposons de l'illustrer sur dix cas relativement représentatifs de la cobotique :

1) Un robot d'assistance à la chirurgie commandé à distance à l'aide d'un écran de visualisation et de manettes pour le pilotage des pinces insérées dans le corps du patient.

2) Un petit drone d'observation télécommandé à distance au moyen d'un joystick et de lunettes d'immersion.

3) Un robot au buste humanoïde commandé à distance : l'humain voit par les yeux du robot et prend possession de ses bras par téléprésence.

4) Un exosquelette facilitant le transport de charges lourdes.

5) Un véhicule à guidage automatique qui reçoit ses ordres d'un opérateur pour transférer une pièce industrielle d'un atelier à un autre, en évitant les obstacles qui sont sur son chemin.

6) Un avion en mode pilotage automatique.

7) Un bras robotique manipulé par l'opérateur et lui permettant de soulever et positionner une pièce lourde devant lui selon une orientation propice à la réalisation de la tâche.

8) Un robot jouet simulant le comportement d'un chien, comportant de nombreux capteurs et effecteurs pour se déplacer en évitant les obstacles.

9) Un robot humanoïde d'accueil capable de dialoguer avec un humain pour le renseigner sur ce qu'il peut trouver dans une exposition.

10) Un bras robotique servant au nettoyage d'une cuve et comportant plusieurs modes d'action, soit en autonomie pour un raclage latéral standard, soit en mode commandé à distance à l'aide d'une interface haptique pour effectuer des mouvements spécifiques (Moulières-Seban 2016).

\subsection{Graphe décrivant l'interaction}

Ces quatre variables peuvent être visualisées en même temps sur un schéma qui synthétise l'interaction et que nous appelons «schème de l'interaction ». Notre objectif est que ce schème puisse aisément correspondre à une représentation mentale de celle-ci. Dans cette description, les 4 grandes parties H, C, E et I sont représentées. Les flux d'informations et les actions sont décrits par des flèches. Lorsque le flux est important (critère subjectif non quantifié dans le cadre de cette étude), l'épaisseur de la flèche est doublée. L'humain et le cobot ayant à traiter des données brutes ou des informations abstraites, nous proposons d'introduire deux sous-parties, la première pour caractériser un traitement de l'information de bas niveau et la deuxième pour caractériser un traitement de haut niveau. Une souspartie est également ajoutée pour la commande motrice. Des exemples de schème sont présentés à la section 4. Cette représentation est intéressante, car elle permet de saisir en un seul coup d'œil des caractéristiques fondamentales de l'interaction. Cette classification permet également de regrouper certains cobots en fonction de caractéristiques similaires de leur schème. Enfin, nous tenterons autant que possible de donner du sens à chaque schème en leur attribuant un nom. 


\section{Caractérisation des schèmes d'interaction}

\subsection{Schème du drone}

Le schème du drone est présenté figure 4.1. La première caractéristique importante est le cheminement de l'information, qui suit un trajet très particulier, de l'environnement au cobot, puis à l'interface, puis à l'humain et qui revient enfin au drone pour l'action sur l'environnement. Il y a ainsi un couplage total entre le cobot et l'humain, avec une dépendance de tous les instants. Il est remarquable que l'humain n'exerce aucune action directe sur l'environnement (difficile pour lui de voler ou d'aider à faire voler) et que le drone n'ait jamais à traiter les données visuelles pour tenter d'un extraire une représentation abstraite. Or, précisément, dans un cas comme dans l'autre, ce sont les points faibles qui sont traités ou mis en œuvre par l'entité collaboratrice. Le schème du drone obéit donc à une logique d'exploitation des informations et d'efficacité de l'action. De façon tout aussi remarquable, il est aisé de montrer que ce schème est également celui du cobot d'assistance pour la chirurgie. Les commandes de vol sont remplacées par les commandes de bras articulés mais les flux d'information sont identiques et l'interface a également un rôle central dans l'interaction. Un autre cobot a un schème similaire à celui du drone, c'est celui de l'humanoïde commandé à distance par téléprésence. C'est en effet une version à peine plus sophistiquée du robot d'assistance chirurgicale. La différence réside surtout dans l'intensité du couplage. Cette différence peut se manifester sur le schème grâce à une épaisseur de trait accentuée pour la relation entre l'humain et l'interface ou entre le cobot et l'interface. En raison du couplage permanent et de la distribution spécifique et efficace des rôles du cobot et de l'humain, à l'instar des premiers travaux de Licklider (LIC 1960), nous proposons le qualificatif de « schème symbiotique ». De légères différences peuvent être observées selon le système cobotique. Il peut en effet être utile de passer par des représentations plus abstraites pour le cobot, afin de prendre en considération des contraintes de la tâche, par exemple en interdisant certains mouvements qui présenteraient des problèmes de sécurité, ou en informant l'opérateur d'une difficulté ou d'un problème dont il ne serait peut-être pas conscient. Dans le cas de la robotique chirurgicale, on peut également noter que le système cobotique ne peut pas remplacer totalement le chirurgien autonome, car la compétence de ce dernier ne peut être acquise que par l'expérience, avec une manipulation directe sans l'entremise du robot.

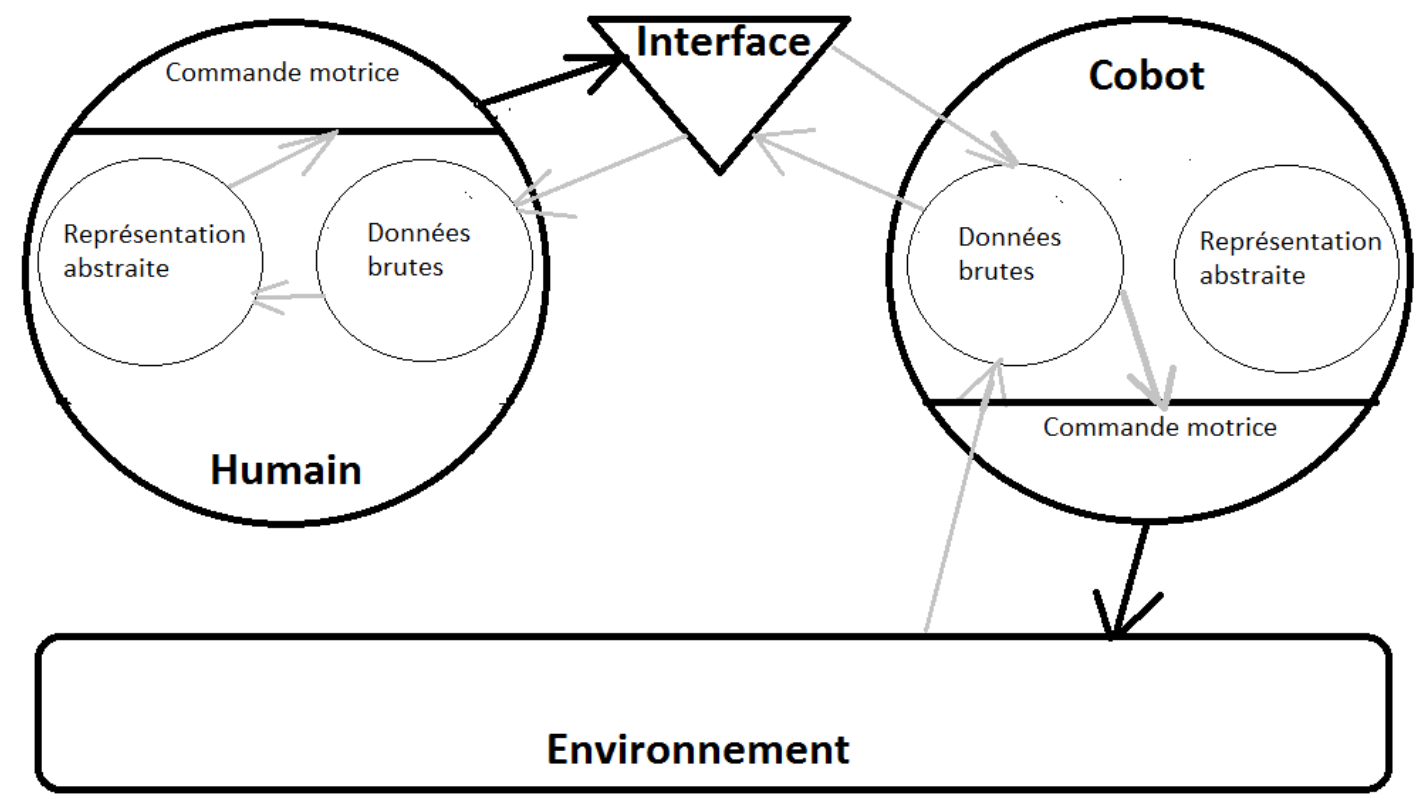

Figure 4.1. Schème d'interaction pour le drone, le robot d'assistance chirurgicale ou le robot humanoïde commandé à distance. Schème symbiotique. 


\subsection{Schème de l'exosquelette}

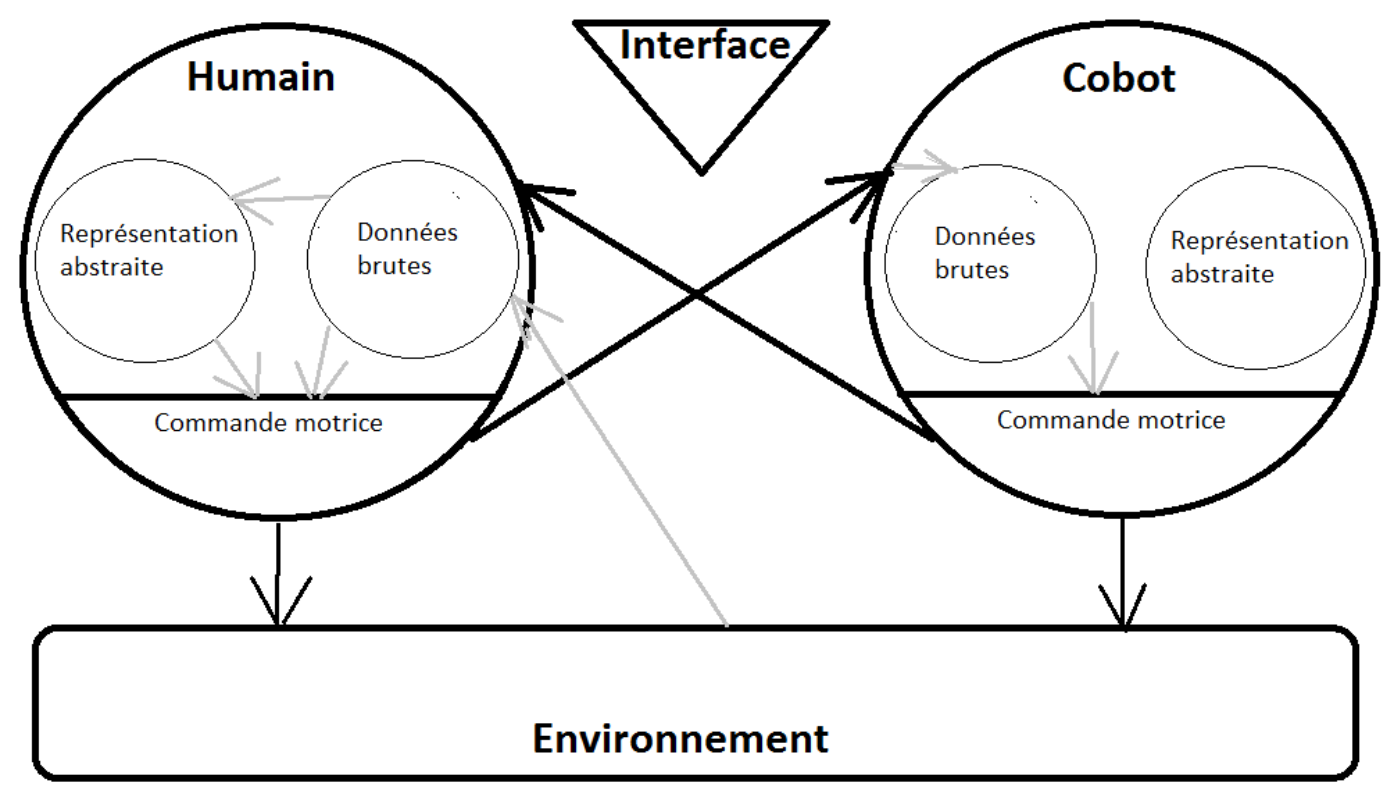

Figure 4.2. Schème d'interaction de l'exosquelette. Schème de l'humain augmenté.

Le schème de l'exosquelette est très particulier. En premier lieu et en première approximation, il n'y a aucune interface entre le cobot et l'humain, le couplage est direct (il existe généralement une interface, mais son rôle est secondaire). Deuxièmement, l'intensité du couplage est élevée (traits forts). La troisième caractéristique est le partage de l'action vis-à-vis de l'environnement. L'objectif étant ici de soulager l'humain de l'effort, l'action du cobot doit être simultanée et proportionnée. On peut noter que le schème n'indique pas le caractère simultané de l'action, ce qui est une des principales lacunes. Autre caractéristique concernant le traitement de l'information, comme pour le drone, on évite les traitements complexes et les représentations complexes, qui sont dévolues à l'humain. Une autre grande spécificité de ce schème est l'absence de prise d'informations sur l'environnement par le cobot. En réalité, le retour de force informe l'exosquelette de son appui au sol, mais il s'agit d'une information directement liée à l'action et à la proprioception, il n'y a en général pas d'autre capteur. Le cobot est donc entièrement focalisé sur l'humain pour l'assister dans ses actions motrices. En raison de l'assistance de tous les instants et de l'intégration au plus près du corps humain visant à en augmenter ses capacités, nous proposons le qualificatif classique de « schème de l'humain augmenté ».

Remarque : dans le cas d'une prothèse, il ne s'agit pas d'accompagner le mouvement d'un bras ou d'une jambe, mais de fournir un membre de substitution. Le couplage est donc moins important, ce qui peut se traduire par un trait atténué. Dans le cadre d'une problématique de réhabilitation fonctionnelle, le couplage est également réduit.

\subsection{Schème du véhicule à guidage automatique}

Différentes options existent pour commander ce type de véhicule et interagir avec lui. Si on se restreint à la première phase d'exploitation, c'est-à-dire à la commande pour que le véhicule se déplace à un autre endroit de l'atelier pour y amener un objet, en évitant les obstacles et éventuellement les humains rencontrés dans la même zone, on obtient un schème totalement asymétrique, qui correspond à celui de la sous-traitance d'une tâche. 


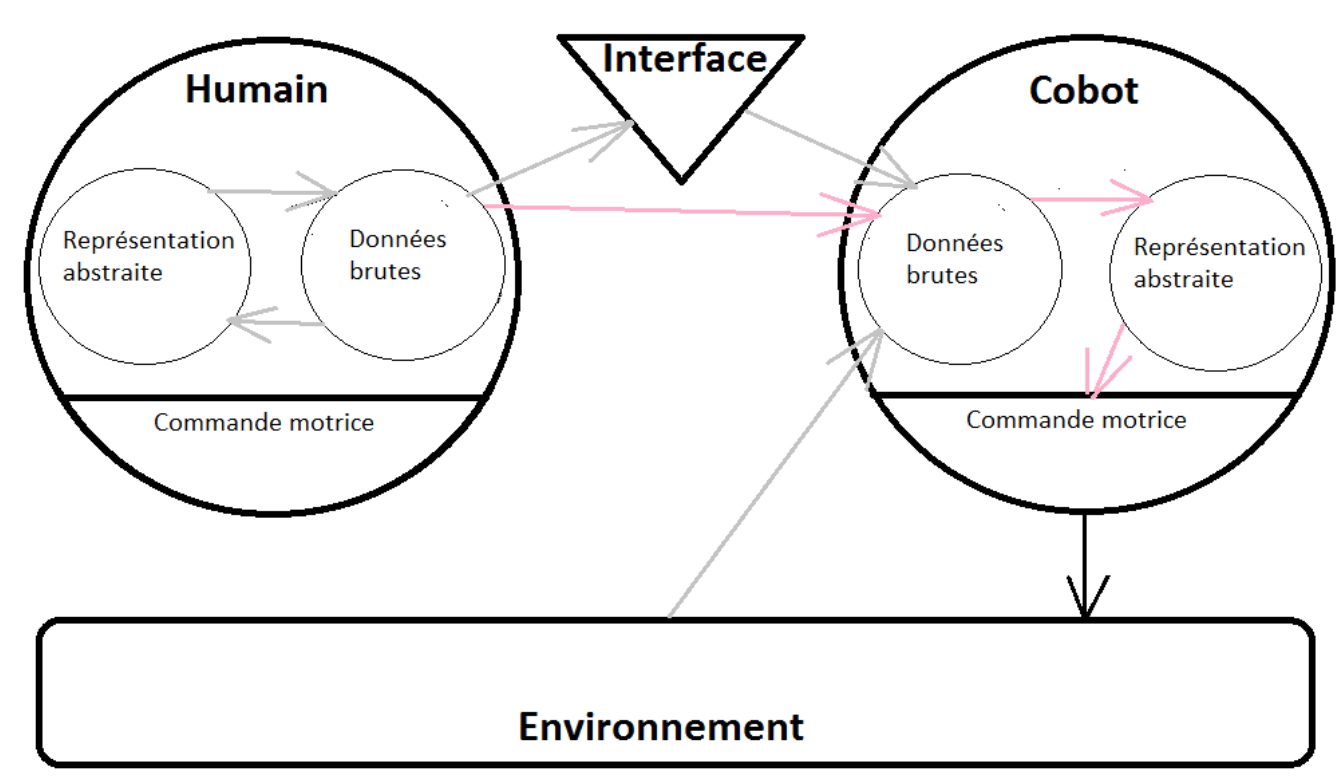

Figure 4.3. Schème d'interaction du véhicule à guidage automatique. Schème de la sous-traitance.

La commande est exécutée par l'humain, soit par une interface, soit directement, en général en appuyant simplement sur quelques boutons. Aucun retour n'est attendu du cobot, qui doit obéir et n'a a priori aucune information à fournir à l'humain lors de cette première phase. Ensuite, le déplacement du véhicule peut être simple à automatiser (par exemple suivi d'une ligne). Les techniques d'intelligence artificielle permettent aujourd'hui des déplacements un peu plus libres, avec une identification des obstacles et la capacité d'effectuer des contournements. Pour cette raison, on peut considérer qu'il y a une représentation complexe de l'environnement. Ce cobot est utile car il permet la sous-traitance d'une tâche pénible, mais la collaboration est limitée. Nous pouvons même considérer que si le déplacement a lieu dans une zone contrôlée où ne figure aucun humain, il s'agit d'un robot classique en train d'exécuter une commande simple. Toutefois, si le robot est capable d'effectuer sa tâche au milieu de l'atelier, dans la même zone que l'humain, l'interaction est plus complexe et la sous-traitance comporte une certaine liberté d'action et de collaboration que n'ont pas les robots classiques. Nous proposons d'appeler ce schème " schème de la sous-traitance ".

Il est aisé de montrer que ce schème est également celui de l'avion en pilotage automatique, les notions de guidage et d'automatisation étant généralisables à d'autres environnements. Le pilote informe le système des paramètres de vol et ce dernier contrôle l'ensemble de l'appareil pour amener l'avion à destination. Il s'agit également d'une sous-traitance, avec une interface plus complexe.

\subsection{Schème du bras robotique d'aide à la manipulation}

Intuitivement, ce cobot est un intermédiaire entre un exosquelette et un cobot sous-traitant. Son schème révèle effectivement qu'il est proche de ces deux catégories. Toutefois, à la différence d'un exosquelette, le cobot n'agit pas sur l'humain, il n'agit que sur l'objet de la tâche. Il est commandé directement par l'humain au moyen d'une force exercée par celui-ci, ou par le biais d'une interface, ou les deux. De même, ce n'est pas de la sous-traitance car l'humain n'est pas dispensé de la tâche, il est aidé dans ses efforts mais il doit également effectuer une manipulation sur l'objet, ce qui se traduit sur le schème par une action de l'humain sur l'environnement. Nous proposons donc le qualificatif de « schème de l'assistance à l'effort ». 


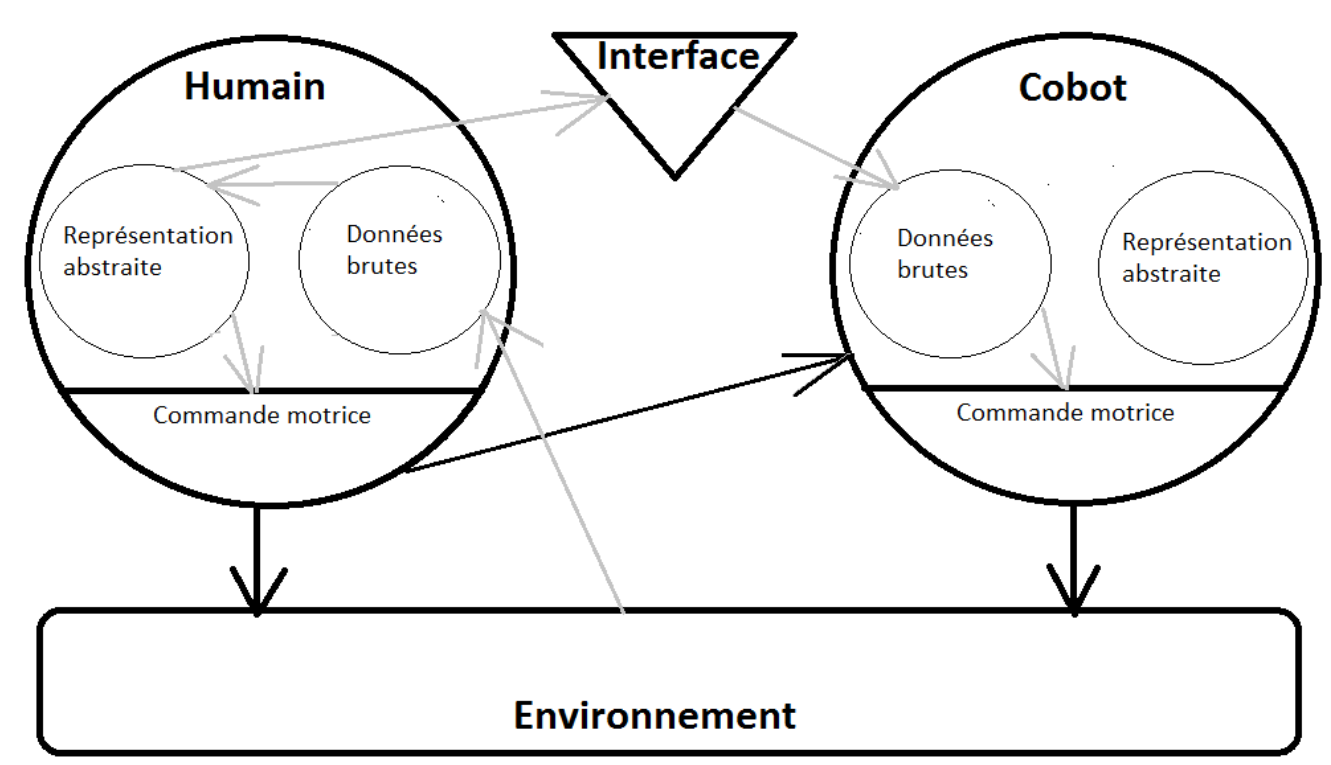

Figure 4.4. Schème d'interaction du bras robotique d'aide à la manipulation. Schème de l'assistance à l'effort.

\subsection{Schème du robot jouet ou du robot d'accueil}

Nous préférons ici parler de robot plutôt que de cobot, car s'il y a bien interaction, il est difficile de parler d'une véritable collaboration. Ces deux types de robot ont la particularité d'interagir de manière complexe avec l'humain. Une de leurs caractéristiques essentielles est d'intégrer un traitement complexe de l'information, notamment pour la reconnaissance de la parole ou la vision. Toutefois, une analyse approfondie suggère que l'interaction est surtout d'ordre informationnelle. Le robot agit sur l'environnement, mais pas sur l'humain, ou de manière superficielle. La partie motrice est souvent assez complexe, avec de nombreux degrés de liberté, mais avec peu de capacité de préhension ou de manipulation. Il y a également de nombreux capteurs, mais le robot n'est pas fait pour travailler avec l'humain. On peut le voir comme un ensemble de deux sous-parties distinctes : la première partie est dédiée à la coordination sensori-motrice pour simuler un comportement animal ou social. La deuxième partie, essentiellement logicielle et relativement indépendante de la première, permet l'interaction « intelligente » avec l'humain. On peut noter que dans le cas du robot humanoïde d'accueil, il n'est même pas besoin que l'humain soit en contact avec la machine (relation en trait discontinu dans le schème de la figure 6).

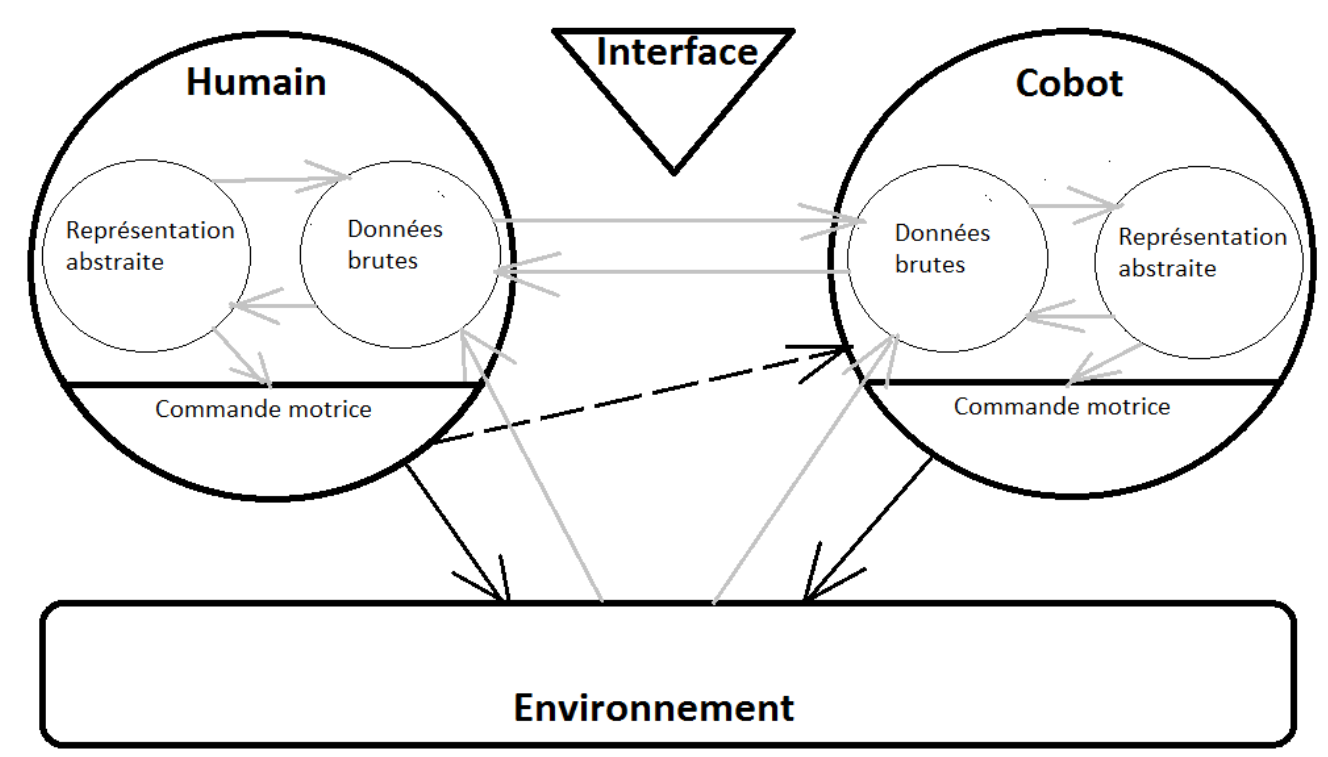

Figure 4.5. Schème d'interaction du robot jouet. Schème de l'assistance intelligente.

Ce système pourrait être qualifié d'ordinateur doté de mécanismes automatiques pour un meilleur design psychocognitif ou sociocognitif, mais la partie mécanique n'a aucune utilité en lien avec la 
collaboration. Pour ce qui est du robot chien, la partie mécanique permet une certaine autonomie de déplacement et de comportement, mais peu d'utilité pratique concernant la collaboration avec l'humain. Des versions plus évoluées permettraient une véritable collaboration, notamment pour la robotique de service. Il reste néanmoins de nombreuses difficultés à surmonter, le robot étant amené à évoluer dans un environnement complexe avec présence d'humains. Tant qu'il reste peu encombrant et sans grande force, sa présence est acceptable et la dangerosité est maitrisée, mais pour qu'il devienne utile, il faudrait qu'il soit plus fort et plus habile, ce qui parait en opposition avec l'acceptabilité, au moins à l'heure actuelle.

Pour conclure sur ces deux types de robot, nous proposons le qualificatif de schème d'assistance cognitive concernant le robot humanoïde d'accueil. Pour le robot jouet, si on y intègre une interface, on est proche du schème d'assistance à l'effort. Aller chercher une balle et la ramener est en effet relativement proche d'une assistance à la tâche. A partir du seul schème, il n'est pas toujours possible de faire une distinction entre une aide à l'effort sur un même objet, ou une aide sur une sous-tâche indépendante. Néanmoins, le fait qu'il y ait un traitement complexe de l'information suggère une certaine aptitude à effectuer une tâche complexe et donc une certaine indépendance. Pour cette raison, nous proposons le qualificatif de schème d'assistance intelligente, qui peut dériver facilement sur le concept de jouet intelligent, ou sur des applications industrielles.

\subsection{Schème du cobot pour le nettoyage d'une cuve}

Ce dernier cobot est intéressant car il illustre la problématique industrielle de développement de cobots là où il est important d'automatiser sans avoir la capacité de le faire de manière totalement indépendante de l'humain, pour des raisons de complexité de la tâche et de variabilité des conditions initiales (MOU 2016). Le cobot est actuellement au stade de prototype testé en environnement simplifié. Pour des raisons de sécurité (produit inflammable), il est commandé à distance via une interface dédiée, avec une caméra montée sur un bras qui est suspendu au-dessus de la grande cuve qu'il faut nettoyer. Un racloir est disposé au bout du bras. Grâce à cette interface, il est possible de réaliser la tâche de nettoyage de manière automatique. Pour cela, le bras positionne le racloir au fond de la cuve et effectue des mouvements radiaux pour amener le produit au centre, là où est situé le trou d'évacuation. Il s'agit clairement d'un mode de sous-traitance, voire d'un mode opératoire de robotique classique. Le robot n'évoluant pas dans la même zone que les humains, le qualificatif de classique parait approprié. Cependant, il y a une supervision de la tâche en temps réel par un opérateur. Si le travail n'est pas parfait, ou s'il faut effectuer une action de raclage particulière, il est possible d'arrêter ce mode opératoire et de commander le bras robotique à l'aide d'un joystick, tout en visualisant l'action grâce à la caméra. Dans ce cas, on retrouve le mode drone et le schème symbiotique, l'action de l'humain et du cobot étant totalement couplée, le premier effectuant la partie pénible et le second le traitement complexe de l'information et la commande complexe. En raison de la supervision et de la possibilité de basculement du mode opératoire, nous préférons qualifier l'action autonome de sous-traitance. On voit donc ici que la solution est un mixte de deux schèmes vus précédemment.

\section{Conclusion}

L'approche que nous avons proposée est basée sur l'étude des flux d'informations entre les différentes parties. Nous avons identifié 5 schèmes qui caractérisent la collaboration homme cobot et qui ont été qualifiés de schème symbiotique, schème de l'humain augmenté, schème de la soustraitance, schème de l'assistance à l'effort, et schème de l'assistance intelligente. Quelques variations existent, mais dans l'ensemble, il s'agit de modes de collaboration différents avec des propriétés clairement identifiées. Cette classification a permis de regrouper plusieurs types de cobot dans une même catégorie, par exemple le drone avec le robot d'assistance chirurgicale et le robot humanoïde commandé à distance. Elle permet également de distinguer des solutions dont la fonction est proche, comme l'exosquelette et le cobot d'aide à la manipulation, qui ont pour but d'aider l'humain à porter et 
manipuler des objets, mais qui ont un schème clairement différencié, le premier étant qualifié d'humain augmenté et le second d'assistance à l'effort.

Cette classification permet d'appréhender la problématique de la cobotique sous un autre angle. En vue d'une application industrielle, il peut s'avérer utile de voir quelles sont les différents types d'interaction et les grands ensembles de solutions. Parfois, comme dans le cas du nettoyage de cuves, plusieurs modes de fonctionnement peuvent être envisagés, sans que cela conduise à de profondes modifications de la solution robotique.

Pour conclure, nous voudrions attirer l'attention sur la solution qualifiée de symbiotique. Il existe en effet un très grand nombre de cas où l'activité hmaine pose problème, parce que l'environnement est dangereux (radiations, produits inflammables, hauteur par rapport au sol, etc.) ou que la tâche est pénible (objets lourds, positions inconfortables, environnement stressant, etc.). La solution de la commande à distance apparait alors naturellement. Si c'est automatisable, la robotisation est à envisager. Cependant, la tâche à accomplir est souvent difficile à automatiser, en raison de la variabilité, de la complexité ou du coût de l'investissement. Dans ce cas, la commande à distance par téléprésence est probablement la plus adaptée. Le schème symbiotique permet la plus grande efficacité pour la réalisation de la tâche. L'inconvénient majeur est l'investissement nécessaire pour développer le robot, le software et l'interface de la solution retenue. On peut cependant noter que dans le cas du buste de robot humanoïde commandé à distance, la prise en mains du robot peut être naturelle. Grâce à sa capacité d'adaptation, l'humain, placé en immersion dans un autre monde, se projette naturellement et avec peu d'efforts cognitifs dans les yeux et les bras du robot. Dans ce cas, la solution pourrait être générique à de nombreuses applications et disponible rapidement. Par exemple, un même robot immersif commandé à distance et à la force décuplée pourrait servir à l'assemblage de pièces lourdes positionnées en hauteur. Ce type de cobot a déjà été développé par la NASA pour effectuer des tâches à l'extérieur de la station spatiale internationale (Robonaut) ou pour l'exploration sous-marine (Ocean One, de Stanford University), mais à notre connaissance, il n'est pas encore exploité dans l'industrie. Ce type de solution pourrait émerger rapidement dans la perspective de l'industrie du futur.

\section{La bibliographie}

[BAR 2004] BARTNECK C. AND Forlizzi J., A Design-Centred Framework for Social Human-Robot Interaction. Proceedings of the Ro-Man2004, Kurashiki pp. 591-594, 2004.

[BIC 2008] Bicchi A., Peshkin M.A., Colgate J.E., Safety for Physical Human-Robot Interaction. In.: Springer Handbook of Robotics (Siciliano, B., Khatib, O.), Springer, Berlin, 2008.

[BRE 2000] BREAzeAl C., Sociable Machines: Expressive Social Exchange Between Humans and Robots, Sc. D. dissertation, Department of Electrical Engineering and Computer Science, MIT (USA), 2000.

[BRO 2004] BROOKS R., Robots are here, MIT Technology Review, 10 Emerging Technologies that will change your world, February 2004.

[BRO 2013] BROOKS R., Robots at Work: Toward a Smarter Factory, Futurist, Vol. 47 Issue 3, p24, may 2013.

[Cla 2013] Claverie B., Le Blanc B. et Fouillat F., "La Cobotique," Presses univ. de Bordeaux | « Communication \& Organisation », vol. $2 n^{\circ}$ 44, pp. 203-214, 2013.

[COL 1996] Colgate J.E., Wannasuphoprasit W., Peshkin M.A., Cobots: Robots for collaboration with human operators, Proc. ASME Dyn. Syst. Cont. Div., Vol.58, p. 433-440, 1996.

[ERD 2012] ERDYN Consultants, Le développement industriel futur de la robotique personnelle et de service en France, rapport publié par le Pôle Interministériel de Prospective et d'Anticipation des Mutations Economiques (PIPAME), Direction Générale de la Compétitivité, de l'Industrie et des Services, avril 2012.

[FER 2007] FerRIS D.P., SAWICKI G.S. AND DALEY M.A., A physiologist's perspective on robotic exoskeletons for human locomotion, International Journal of Humanoid robotics, vol. 4, p. 507-528, 2007.

[FER 2009] FERRIS D.P., The exoskeletons are here, Journal of NeuroEngineering and Rehabilitation, Commentary, vol. 6 (17), June 2009. 
[HAD 2007] HADDADIN S., ALBU-SCHAEFFER A., HIRZINGER G., Safe Physical Human-Robot Interaction: Measurements, Analysis and New Insights. In Proceedings of the 13th International Symposium of Robotics Research (ISRR2007). Hiroshima, Japan, November 26-29, 2007.

[HER 2009] HERR H., Exoskeletons and orthoses: classification, design challenges and future directions, Journal of Neuroengineering and Rehabilitation, vol. 6 (21), June 2009.

[HON 2007] HONDA COMPANY, Asimo, Technical Information, http://asimo.honda.com/downloads/, September 2007.

[LEE 2014] LEE N., « Robotic surgery: where are we now? », Lancet, Volume 384, No. 9952, p1417, 18 October 2014.

[LEM 2015] Lemaignan S., Warnier M., Sisbot E. AND Alami R., Human-Robot Interaction: Tackling the AI Challenges, Artificial Intelligence, January 2015.

[LIC 1960] LICKLIDER J.C., Man computer symbiosis, IRE Transactions on Human Factors in Electronics, volume HFE1, pages 4-11, March 1960.

[MOU 2015] Moulières-Seban T., Salotti J.M., Claverie B. And Bitonneau D., Classification of cobotic systems for industrial applications, proceedings of the $6^{\text {th }}$ workshop towards a Framework for Joint Action, Paris (France), October 26th, 2015 (available at: https://fja.sciencesconf.org/conference/fja/Moulieres.pdf).

[MOU 2016] Moulières-Seban T. Bitonneau D., Salotti J.M., Thibault J.F. And Claverie B., Human Factors Issues for the Design of a Cobotic System, Proceedings of the AHFE 2016 International Conference on Human Factors in Robots and Unmanned Systems, Walt Disney World®, Florida, USA, July 27-31, 2016.

[PAR 2000] PARASURAMAN R.AND SHERIDAN T.B., A Model for Types and Levels of Human Interaction with Automation. IEEE Transactions on Systems, Man, and Cybernetics - Part A: Systems and Humans, vol. 30 (3), 2000.

[PES 1999] PeshKin M., Colgate J., Cobots, Industrial Robot: An International Journal, Vol. 26 (5), pp. 335-341, 1999.

[RAH 1990] RAHIMI M. AND KARWOWSKI W., A research paradigm in human robot interaction, International Journal of Industrial Ergonomics (Elsevier), vol. 5, p. 59-71, 1990.

[RUS 2016] RUSSEl S. AND NORVIG P., Artificial Intelligence, A Modern Approach, $3^{\text {rd }}$ edition, Pearson ed., 2016.

[SCH 2003] SCHOLTZ J., Theory and Evaluation of Human Robot Interactions. Proc. of the $36^{\text {th }}$ Hawaii International Conference on System Sciences (HICSS'03), 2003.

[SHE 2016] SHERIDAN T.B., Human-Robot Interaction: Status and Challenges, Human Factors, vol. 58 (4), June 2016.

[SMA 2013] « Industries 4.0, Le Coeur des Usines Intelligentes », Smart Industries, septembre 2013.

[SON 2001] SONY CORPORATION, Entertainment Robot AIBO, Operating Instructions ERS-210, 2001.

[THR 2005] Thrun S., BURGard W. AND Fox D., Probabilistic Robotics, MIT Press, 2005.

[WAL 2014] WALther S., GUHL T., Classification of physical human-robot interaction scenarios to identify relevant requirements, proceedings of the $45^{\text {th }}$ International Symposium on Robotics and $8^{\text {th }}$ German Conference on Robotics (ISR/ROBOTIK), session "Human-Robot Interaction”, June 2014.

[WOO 1999] WoOlRIDGe M., Multiagent Systems. A Modern Approach to Distributed Artificial Intelligence., Massachusetts Institute of Technology, 1999.

[YAN 2004] YANCO H.A. AND DRURY J., Classifying Human-Robot Interaction: An Updated Taxonomy, 2004. 